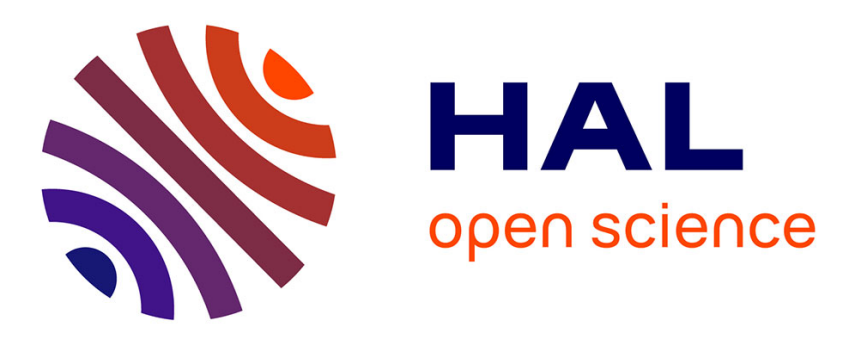

\title{
Non-linear source separation under the Langmuir model for chemical sensors
}

\author{
Pierre Maho, Simon Barthelme, Pierre Comon
}

\section{To cite this version:}

Pierre Maho, Simon Barthelme, Pierre Comon. Non-linear source separation under the Langmuir model for chemical sensors. SAM 2018 - 10th IEEE Workshop on Sensor Array and Multichannel Signal Processing, Jul 2018, Sheffield, United Kingdom. pp.380-384, 10.1109/SAM.2018.8448636 . hal-01802358

\section{HAL Id: hal-01802358 \\ https://hal.science/hal-01802358}

Submitted on 29 May 2018

HAL is a multi-disciplinary open access archive for the deposit and dissemination of scientific research documents, whether they are published or not. The documents may come from teaching and research institutions in France or abroad, or from public or private research centers.
L'archive ouverte pluridisciplinaire HAL, est destinée au dépôt et à la diffusion de documents scientifiques de niveau recherche, publiés ou non, émanant des établissements d'enseignement et de recherche français ou étrangers, des laboratoires publics ou privés. 


\title{
Non-linear source separation under the Langmuir model for chemical sensors
}

\author{
Pierre Maho, Simon Barthelmé, Pierre Comon \\ Univ. Grenoble Alpes, CNRS, Grenoble INP, Gipsa-Lab, 38000 Grenoble, France \\ firstname.lastname@gipsa-lab.grenoble-inp.fr
}

\begin{abstract}
Electronic nose is a promising bio-inspired instrument for the detection of Volatil Organic Compounds (VOCs), meaning a compound containing carbon which easily evaporates. One of the most important parts of these devices is a set of non-specific chemical sensors, which will interact with the VOC and output valuable information for its identification. The nonspecificity of these chemical sensors ensures the universality of the instrument. The main task achieved by this instrument is the detection of individual VOC. However, in many real-life applications, mixtures of VOCs are observed. The recovery of the mixture composition, meaning the individual signatures and their relative contribution, is a challenging task which can be studied in a Blind Source Separation framework. In this paper, we propose a non-linear mixture model for a particular type of chemical sensors. This model is based on the Langmuir isotherm for a multi-component gas. We study the joint identifiability of signatures and concentrations, and propose a necessary identification condition. Finally, we propose an algorithm for the blind estimation of the parameters and assess its performance through simulations.
\end{abstract}

Index Terms-non-linear source separation, Langmuir, electronic nose, two-block coordinate descent, gas mixture

\section{INTRODUCTION}

Electronic nose (eNose) is a bio-inspired instrument which is able to detect and identify Volatile Organic Compounds (VOCs). A VOC is a chemical compound which contains carbon and can be easily evaporated. An eNose is composed of several non-specific chemical sensors and a transduction method [1]. First, the VOC interacts with the set of chemical sensors, providing chemical information on the VOC, which is assumed to be highly VOC-dependent. Then, the transduction method transforms the chemical information into a usable one such as electrical or optical. The outputs are time-series from which features are extracted in order to create a signature for a given VOC.

The chemical sensors of an eNose are expected to be nonspecific, meaning that they can interact with a broad variety of VOCs. This non-specificity is in fact a requirement for having a universal device [2]. In a real-life application, the instrument can be exposed to mixtures of several VOCs. In this case, the resulting output will be no more the individual signature of a VOC but the mixing of all the individual signatures.

Although it is clearly a Blind Source Separation (BSS) case, this issue has been ignored by the literature of eNoses. To the authors' knowledge, the issue of BSS for eNoses was raised only recently [3].

This work was supported in part by ERC Advanced Grant 2013-320594 DECODA, FUI WISE and ANR Gen GP.
This article tries to solve BSS problem for a type of chemical sensors used for an eNose. First, we describe a theoretical non-linear mixture model based on the Langmuir isotherm [4] for a multi-component gas. Then, some theoretical results on identifiability in the blind case are provided. We propose an algorithm based on a two-block coordinate descent in order to recover the sources and their relative contributions. Finally, their reconstruction is validated through simulations.

\section{PROBlem STATEMENT}

The chemical sensors studied in this contribution are those recently described in [5]. Briefly, the sensitive layer of the sensor consists of a biomolecular receiver, called a ligand, which is dropped off a golden surface. Thanks to an air flow, the analysed VOC, called an analyte, is brought above the surface and interacts with the ligand through a reversible binding reaction. This chemical reaction is recorded in realtime thanks to a Surface Plasmon Resonance imaging (SPRi) technique. Briefly, the surface is illuminated by a monochromatic light which is reflected towards a photodetector. The chemical interactions occurring at the surface change the medium, which in turns modifies the refractive index. Finally, the refractive index changes the reflected light. Consequently, the amount of received light accounts for the binding reaction between the ligand and the analyte. The above explanations are summarized in Figure 1.

To describe the Langmuir model for a multi-component gas, some fundamental assumptions need to be made:

A1 Environmental parameters (temperature, ...) are constant.

A2 There is no interaction between the $R$ analytes.

A3 The binding reaction is only mono-layer.

A4 A binding site can be occupied only by one analyte.

A5 The concentration $c_{r}$ of each analyte is constant during all the injection phase.

\section{A. Model}

1) Single sensor: Let's consider a complex mixture of $R$ analytes measured by the sensor. From Assumptions A1-4, each of the $R$ analytes will interact with the sensitive layer by a binding reaction. Considering all reactions as independent of each other, there are $R$ chemical reactions occurring on the surface:

$$
\mathcal{A}_{r}+\mathcal{L} \underset{d_{r}}{\stackrel{a_{r}}{\rightleftharpoons}} \mathcal{A}_{r} \mathcal{L}
$$

where $\mathcal{A}_{r}, \mathcal{L}, a_{r}$ and $d_{r}$ respectively stand for the $r^{\text {th }}$ analyte, the ligand, the absorption and desorption constants, which are 


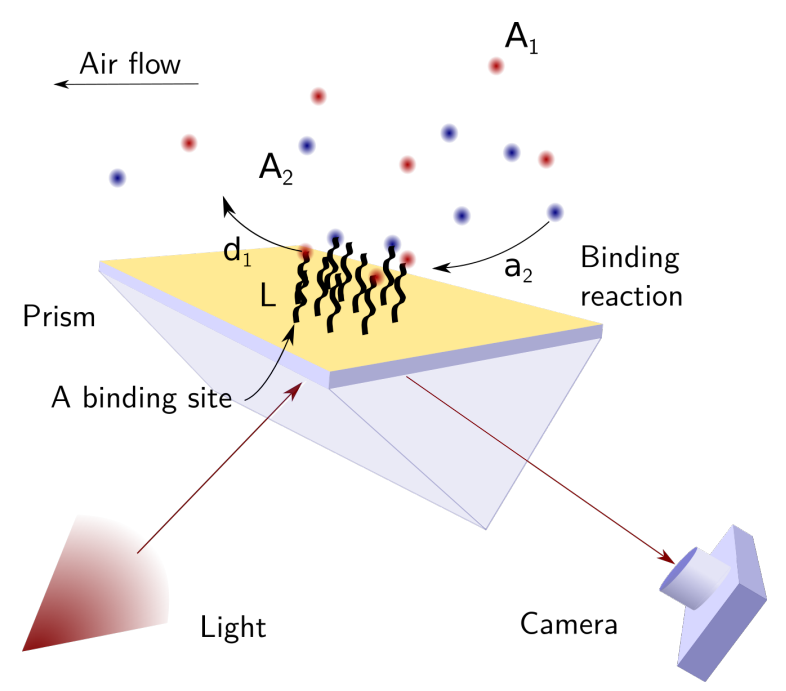

Figure 1: A binary mixture composed of the analytes $\mathcal{A}_{1}$ (red dots) and $\mathcal{A}_{2}$ (blue dots) is injected by air flow above the chemical sensor $\mathcal{L}$. The binding reactions governed by the parameters $\left(a_{r}, d_{r}\right)$ are recorded in real-time thanks to a SPRi technique. See the text for more details.

specific to the reaction. Under assumption A5, we can describe the rate of complex formed $\mathcal{A}_{r} \mathcal{L}$ by the differential equation:

$$
\frac{\mathrm{d}\left[\mathcal{A}_{r} \mathcal{L}\right]_{s}(t)}{\mathrm{d} t}=a_{r} c_{r}\left([\mathcal{L}]_{s}-\sum_{i=1}^{R}\left[\mathcal{A}_{i} \mathcal{L}\right]_{s}(t)\right)-d_{r}\left[\mathcal{A}_{r} \mathcal{L}\right]_{s}(t)
$$

where $[\cdot]_{s}$ stands for the surfacic concentration (in $\mathrm{mol} / \mathrm{m}^{2}$ ). The equation (1) can be simplified to:

$$
\frac{\mathrm{d} \theta_{i}(t)}{\mathrm{d} t}=a_{i} c_{i}\left(1-\sum_{p=1}^{I} \theta_{p}(t)\right)-d_{i} \theta_{i}(t)
$$

where $\theta_{r}(t)=\frac{\left[\mathcal{A}_{r} \mathcal{L}\right]_{s}}{[\mathcal{L}]_{s}}(t)$ stands for the fraction of sites being occupied by the $r^{\text {th }}$ analyte. If we wait for a sufficiently long time $t_{e q}$, the system of chemical reactions will reach an equilibrium phase, meaning that $\forall r, \forall t \geqslant t_{e q}$,

$$
\frac{\mathrm{d} \theta_{r}(t)}{\mathrm{d} t}=0=a_{r} c_{r}\left(1-\sum_{i=1}^{R} \theta_{i}^{e q}\right)-d_{r} \theta_{r}^{e q}
$$

The solution of the $r^{\text {th }}$ equation can be easily obtained by summing the $\mathrm{R}$ equations and isolating $\sum_{i=1}^{R} \theta_{i}^{e q}$. This leads to:

$$
\theta_{r}^{e q}=\frac{k_{r} c_{r}}{1+\sum_{i=1}^{R} k_{i} c_{i}}
$$

where $k_{r}=\frac{a_{r}}{d_{r}} \geqslant 0$. Equation (3) is referred to as the Langmuir isotherm [4] for a multi-component gas.

The SPRi measurement of the described reactions can be assumed to be proportional to the fraction of occupied sites by considering that the medium $\mathcal{A}_{r} \mathcal{L}$ is in the nanometer range [6], [7]. The coefficient of proportion $\alpha$ is then dependent on the SPR settings through a constant $\beta$, the ligand surfacic concentration $[\mathcal{L}]_{s}$ and the mass analyte $m_{r}: \alpha=\beta[\mathcal{L}]_{s} m_{r}$. To simplify, $\beta[\mathcal{L}]_{s}$ is assumed here to equal 1 .
The assumption of a thin layer at the surface also implies that the medium will be viewed as homogeneous by the SPRi. We can then assume that the individual optical outputs will add up:

$$
y=\frac{\sum_{r=1}^{R} k_{r} m_{r} c_{r}}{1+\sum_{r=1}^{R} k_{r} c_{r}}
$$

where $y$ denotes the measurement.

2) Sensor array: At this stage, considering that only $k_{r}$ and $c_{r}$ are unknown, we have $2 R$ parameters and only 1 equation. It is obviously impossible to estimate them correctly with so little information. A classical approach is to consider several sensors, say $P$, with different types of ligands to add equations to the problem. By comparison to the above developments, $k_{r}$ is now written as $k_{p r}$ where the subscript $p$ stands for the sensor index number. The vector $\boldsymbol{k}_{r} \in \mathbb{R}^{P}$ can be seen as the fingerprint - or the olfactive signature - of the analyte $r$. All the individual signatures are stacked in a matrix $\boldsymbol{K}=$ $\left[\boldsymbol{k}_{1}, \ldots, \boldsymbol{k}_{P}\right] \in \mathbb{R}^{P \times R}$.

3) Several mixtures: Now, we have $R(1+P)$ parameters for only $P$ equations. There is still too little information for solving the problem of estimating $k_{p r}$ and $c_{r}$. An other intuitive way to add equations is to consider several experiments, say $N$, of the same mixture but at different individual concentrations. We denote by $c_{r n}$ the concentration of the $r^{\text {th }}$ analyte in the $n^{\text {th }}$ experiment. All the concentration vectors $\mathbf{c}_{n} \in \mathbb{R}^{R}$ are stacked in a matrix $\boldsymbol{C}=\left[\boldsymbol{c}_{1}, \ldots, \boldsymbol{c}_{N}\right] \in \mathbb{R}^{R \times N}$.

4) Final model: Taking into account the sensor dimension (subscript $p$ ) and the experiment dimension (subscript $n$ ), the measurement should now be denoted $y_{p n}$, meaning the output of the $p^{\text {th }}$ sensor for the $n^{\text {th }}$ mixture. As above, we stack all the measurements in a matrix called $\boldsymbol{Y}=\left[\boldsymbol{y}_{1}, \ldots, \boldsymbol{y}_{N}\right] \in \mathbb{R}^{P \times N}$.

Finally, the model can be seen in a scalar form as in (3), but we can now also write it in compact form:

$$
\mathscr{L}(\boldsymbol{K}, \boldsymbol{C})=\boldsymbol{Y}=\boldsymbol{K} \boldsymbol{M C} \square\left(\mathbf{1}_{P} \mathbf{1}_{N}^{\mathrm{t}}+\boldsymbol{K} \boldsymbol{C}\right)
$$

where $M \in \mathbb{R}^{R \times R}$ stands for the diagonal matrix of the masses and $\square$ for the Hadamard (i.e. entrywise) division.

\section{B. Some limiting cases}

The above model can be related to well-known models in the BSS literature in some limiting cases.

1) Low concentration: When we deal with an application where low concentrations are expected, this leads to a quite simpler version of the model (5). Indeed, assuming $C \ll 1$ and $\boldsymbol{K} \approx O(1)$, we can simplify (5) by a 0 -order Taylor expansion:

$$
Y \approx K M C
$$

Then (6) is a linear mixing model, whose identifiability conditions have been addressed in the BSS literature [8], [9].

2) Saturation: At the opposite end, if we work with really high concentrations or with analytes having a really high affinity with the sensor array, then $\boldsymbol{K} \boldsymbol{C} \gg 1$ and (5) gets harder:

$$
\boldsymbol{Y} \approx \boldsymbol{K} M C \square K C
$$


Eq. (7) is clearly non identifiable as $\forall\left(\boldsymbol{D}_{1}, \boldsymbol{D}_{2}\right) \in \mathbb{R}^{P \times P} \times$ $\mathbb{R}^{N \times N}, \boldsymbol{D}_{1} \boldsymbol{K} \boldsymbol{M C D _ { 2 }} \square \boldsymbol{D}_{1} \boldsymbol{K} C \boldsymbol{D}_{2}=\boldsymbol{K M C} \square \boldsymbol{K} \boldsymbol{C}$ where $\left(\boldsymbol{D}_{1}, \boldsymbol{D}_{2}\right)$ are diagonal. Fortunately, this version of (5) is unlikely to appear since it would be useless to put the sensor in a saturation regime.

3) Analyte with the same mass: As a final limiting case, consider analytes having the same mass $m$, i.e. $\boldsymbol{M}=m \boldsymbol{I}$ :

$$
\frac{1}{m} \boldsymbol{Y}=\boldsymbol{K} \boldsymbol{C} \square\left(\mathbf{1}_{P} \mathbf{1}_{N}^{\mathrm{t}}+\boldsymbol{K} \boldsymbol{C}\right)
$$

This particular model falls into a class known as Post NonLinear mixtures [10]. The component-wise inversion $g(y)=$ $\frac{y}{1-y}$ enables to recover the linear model $\tilde{\boldsymbol{Y}}=\boldsymbol{K} \boldsymbol{C}$. Model (8) is interesting but rather unrealistic in an application prospect, as it considerably constrains the set of analytes that can be mixed together.

4) Conclusion: The above special cases of model (5) constrain the range of applications of these chemical sensors for an electronic nose. Therefore, in the following, we will try to deal with the general case.

\section{METHOD}

In this section, the unsupervised - or "blind"- case is studied, meaning that neither $\boldsymbol{K}$ or $\boldsymbol{C}$ are known so that they have to be estimated jointly. The masses $M$ are assumed to be known in order to simplify the study.

\section{A. Permutation issue}

A usual trivial indetermination in a BSS framework is the source permutation, when the model is linear. It is interesting to note that model (5) doesn't suffer from this indetermination, subject to a weak assumption. Indeed, let $Q \in \mathbb{R}^{R \times R}$ be an invertible matrix, and keeping in mind that $M$ is diagonal, we have:

$$
\begin{array}{lcccc} 
& & \mathscr{L}\left(\boldsymbol{K} \boldsymbol{Q}, \boldsymbol{Q}^{-1} \boldsymbol{C}\right) & = & \mathscr{L}(\boldsymbol{K}, \boldsymbol{C}) \\
\Leftrightarrow & & \boldsymbol{Q} \boldsymbol{M} & = & \boldsymbol{M} \boldsymbol{Q} \\
\Leftrightarrow \quad \forall i, j & (\boldsymbol{Q} \boldsymbol{M})_{i j} & = & (\boldsymbol{M} \boldsymbol{Q})_{i j} \\
\Leftrightarrow \quad \forall i, j & \sum_{r=1}^{R} q_{i r} m_{r j} & = & \sum_{r=1}^{R} m_{i r} q_{r j} \\
\Leftrightarrow \quad \forall i, j & q_{i j} m_{j j} & = & m_{i i} q_{i j} \\
\Leftrightarrow \quad \forall i, j & q_{i j}=0 & \text { or } & m_{j j}=m_{i i}
\end{array}
$$

Thus, if we assume that each analyte of the mixture has a different mass, meaning that $\forall i \neq j, m_{j j} \neq m_{i i}$, then the only trivial indetermination is the scaling ambiguity.

\section{B. Identifiability}

Identifiability is generally not a trivial question meaning that the parameters $(\boldsymbol{K}, \boldsymbol{C})$ which have generated the measures $\boldsymbol{Y}$ according to model (5) can be exactly retrieved or not. The study carried out in Section III-A showed that the model will be at best identifiable up to a multiplicative diagonal matrix. Thereafter, identifiability will mean identifiability up to a diagonal matrix.

We assume that we have more sensors and more mixtures than there are analytes, so that $P \geqslant R$ and $N \geqslant R$. To find identification conditions for $C$, we rewrite model (5) noticing that it is separable across experiments:

$$
\begin{aligned}
(5) & \Longleftrightarrow \forall n, \quad \boldsymbol{y}_{n} \cdot\left(\mathbf{1}_{P}+\boldsymbol{K} \boldsymbol{c}_{n}\right)=\boldsymbol{K} \boldsymbol{M} \boldsymbol{c}_{n} \\
& \Longleftrightarrow \forall n, \quad \boldsymbol{y}_{n}=\left(\boldsymbol{K} \boldsymbol{M}-\operatorname{diag}\left(\boldsymbol{y}_{n}\right) \boldsymbol{K}\right) \boldsymbol{c}_{n}
\end{aligned}
$$

Lemma 1. Let $\boldsymbol{A}_{n} \stackrel{\text { def }}{=} \boldsymbol{K} \boldsymbol{M}-\operatorname{diag}\left(\boldsymbol{y}_{n}\right) \boldsymbol{K}$. Then $\boldsymbol{c}_{n}$ is identifiable if and only if $\boldsymbol{A}_{n}$ is full rank. Similarly, let $\boldsymbol{B}_{p} \stackrel{\text { def }}{=} \operatorname{diag}\left(\boldsymbol{y}_{p}\right) \boldsymbol{C}^{t}-\boldsymbol{C}^{t} \boldsymbol{M}$. Then $\boldsymbol{k}_{p}$ is identifiable if and only if $\boldsymbol{B}_{p}$ is full rank.

Proof. The proof is straightforward: assume there are two solutions $\boldsymbol{c}_{n}$ and $\boldsymbol{c}_{n}^{\prime}$. Then $\boldsymbol{A}_{n} \boldsymbol{c}_{n}=\boldsymbol{y}_{n}$ and $\boldsymbol{A}_{n} \boldsymbol{c}_{n}^{\prime}=\boldsymbol{y}_{n}$. By subtraction, $\boldsymbol{A}_{n}\left(\boldsymbol{c}_{n}-\boldsymbol{c}_{n}^{\prime}\right)=\mathbf{0}_{P}$. Since $\boldsymbol{A}_{n}$ is full rank, we have necessarily $\left(\boldsymbol{c}_{n}-\boldsymbol{c}_{n}^{\prime}\right)=\mathbf{0}_{P}$. The proof for $\boldsymbol{k}_{p}$ is the same.

Proposition 1 (Necessary condition). If $\boldsymbol{c}_{n}$ (resp. $\boldsymbol{k}_{p}$ ) are identifiable, then $\boldsymbol{K}$ (resp $\boldsymbol{C}$ ) is full rank, or $\boldsymbol{M}$ is not proportional to the Identity matrix.

Proof. Let's prove the statement for $\boldsymbol{K}$, since a dual reasoning holds for $\boldsymbol{C}$. The proof is by contradiction. Assume $\operatorname{rank}\{\boldsymbol{K}\} \neq R$ and $\boldsymbol{M} \propto \boldsymbol{I} \mathbf{d}$, then $\exists \boldsymbol{v} \in \mathbb{R}_{*}^{R}$ such that $\boldsymbol{K} \boldsymbol{v}=\mathbf{0}_{P}$. Hence $\operatorname{diag}\left(\boldsymbol{y}_{n}\right) \boldsymbol{K} \boldsymbol{v}=\mathbf{0}_{P}$. On the other hand, because $\boldsymbol{M} \propto \boldsymbol{I d}, \boldsymbol{K} \boldsymbol{M} \boldsymbol{v}=\mathbf{0}_{P}$. Hence $\boldsymbol{A}_{n} \boldsymbol{v}=\mathbf{0}_{P}$, which prevents identifiability from Lemma 1.

\section{Algorithm}

In order to retrieve the parameters $(\boldsymbol{K}, \boldsymbol{C})$, we propose to use an alternating least squares algorithm, also called two-block coordinate descent. It consists of minimizing the cost function $\Upsilon$ (see below) with respect to $\boldsymbol{K}$ using an estimation of $\boldsymbol{C}$, and then minimizing w.r.t. $\boldsymbol{C}$ using the previously estimated value of $\boldsymbol{K}$. This process is repeated until convergence.

$$
\Upsilon(\boldsymbol{K}, \boldsymbol{C})=\|\boldsymbol{Y}-\mathscr{L}(\boldsymbol{K}, \boldsymbol{C})\|_{F}
$$

As shown in section II-B, when the product $\boldsymbol{K C}$ is too large compared to 1 , the model can be simplified into a nonidentifiable one, for which there is no hope of recovering the true parameters. By assuming that the latter are not located in this region and to prevent the algorithm from getting stuck in this "non-identifiable area", we propose to regularize it with the max-norm.

\begin{tabular}{l}
\hline \multicolumn{1}{c}{ Algorithm 1: Estimate $(\mathbf{K}, \mathbf{C})$} \\
\hline Require: $\boldsymbol{C}_{0}, \Omega, \epsilon, p_{\max }$ \\
while $\left|\Upsilon\left(\boldsymbol{K}_{p+1}, \boldsymbol{C}_{p+1}\right)-\Upsilon\left(\boldsymbol{K}_{p}, \boldsymbol{C}_{p}\right)\right|>\epsilon \& p<p_{\max }$ \\
do \\
$\quad \boldsymbol{K}_{p+1} \leftarrow \arg \min _{\boldsymbol{K} \geqslant 0,\|\boldsymbol{K}\|_{\max } \leqslant \frac{\Omega}{\left\|\boldsymbol{C}_{p}\right\|_{\max }} \Upsilon} \Upsilon\left(\boldsymbol{K}, \boldsymbol{C}_{p}\right)$ \\
$\quad \boldsymbol{C}_{p+1} \leftarrow \arg \min _{\boldsymbol{C} \geqslant 0,\|\boldsymbol{C}\|_{\max } \leqslant \frac{\Omega}{\left\|\boldsymbol{K}_{p+1}\right\|_{\max }}} \Upsilon\left(\boldsymbol{K}_{p+1}, \boldsymbol{C}\right)$ \\
$\quad p \leftarrow p+1$ \\
end while \\
return $\boldsymbol{K}_{p}, \boldsymbol{C}_{p}$
\end{tabular}


Imagine that we are in the $\boldsymbol{K}$ estimation block, so we have an estimation $\hat{\boldsymbol{C}}$ for $\boldsymbol{C}$. An intuitive way for constraining the amplitude of the product $\boldsymbol{K C}$ is to upper-bound the max-norm with a parameter $\Omega$ :

$$
\forall i, j(\boldsymbol{K} \hat{\boldsymbol{C}})_{i j} \leqslant\|\boldsymbol{K} \hat{\boldsymbol{C}}\|_{\max } \stackrel{\text { def }}{=} R \sup _{i j}\left(\left|(\boldsymbol{K} \hat{\boldsymbol{C}})_{i j}\right|\right) \leqslant \Omega
$$

Clearly, constraint (10) would not be trivial to implement. So, we relax it by noticing that the max-norm $\|\cdot\|_{\max }$ is submultiplicative [11]:

$$
\|\boldsymbol{K} \hat{\boldsymbol{C}}\|_{\max } \leqslant\|\boldsymbol{K}\|_{\max }\|\hat{\boldsymbol{C}}\|_{\max } \leqslant \Omega
$$

Now constraint (11) imposes that the maximum value of $\boldsymbol{K}$ is lower than or equal to $\frac{\Omega}{\|\hat{\boldsymbol{C}}\|_{\max }}$. Thus, it can be easily implemented in addition to the non-negativity constraint as a box-constraint. For the minimization of each block, we propose to use the L-BFGS method [12], which can easily incorporate box-constraints.

\section{COMPUTER RESUlts}

To validate Algorithm 1 and assess its performance in the presence of noise, we carry out simulations. The number of sensors, $P$, is set to 100 and the number of experiments, $N$, to 100 . We vary the number of analytes in the mixtures, $R$, to 5,10 and 15 . The vector of masses is $[10,20, \ldots, 140,150]^{\mathrm{t}}$ $\mathrm{g} / \mathrm{mol}$ (we take the 5 first masses when $R=5$, etc...) and $\Omega=$ 100. Matrices $\boldsymbol{K}$ and $\boldsymbol{C}$ are generated randomly according to an exponential law with $\lambda=1.5$. The noise is additive and Gaussian with zero-mean and standard deviation $\sigma_{n}$. When negative values are created due to noise addition, we clip them to 0. $\sigma_{n}$ depends on the Signal to Noise Ratio (SNR) defined as:

$$
\mathrm{SNR}=20 \log \left(\frac{\sigma_{s}}{\sigma_{n}}\right) \text { with } \sigma_{s}=\sqrt{\frac{\|\boldsymbol{Y}\|_{F}}{P N}}
$$

As shown in section III, parameters $(\boldsymbol{K}, \boldsymbol{C})$ are identifiable up to a diagonal matrix when the masses are different for each analyte. Consequently, to assess estimations $(\hat{\boldsymbol{K}}, \hat{\boldsymbol{C}})$, a correlation measure is well suited:

$\operatorname{Cor}_{\boldsymbol{K}}=\frac{1}{R} \sum_{r=1}^{R} \operatorname{cor}\left(\boldsymbol{k}_{r}, \hat{\boldsymbol{k}}_{r}\right) \quad \operatorname{Cor}_{\boldsymbol{C}}=\frac{1}{R} \sum_{r=1}^{R} \operatorname{cor}\left(\boldsymbol{c}_{r}, \hat{\boldsymbol{c}}_{r}\right) \quad$ with, $\forall(\boldsymbol{x}, \boldsymbol{y}) \in \mathbb{R}^{p}, \operatorname{cor}(\boldsymbol{x}, \boldsymbol{y})=\frac{\sum_{i=1}^{p}\left(x_{i}-\bar{x}\right)\left(y_{i}-\bar{y}\right)}{\sqrt{\sum_{i=1}^{p}\left(x_{i}-\bar{x}\right)^{2}} \sqrt{\sum_{i=1}^{p}\left(y_{i}-\bar{y}\right)^{2}}}$ where ${ }^{-}$stands for the mean of the vector.

Even if the model doesn't suffer theoretically from the permutation issue, in practice some local minima can correspond to permuted sources. This problem is overcome by taking each column of $\hat{\boldsymbol{K}}$ (resp. $\hat{\boldsymbol{C}}^{\mathrm{t}}$ ) in an arbitrary order. We associate it with the closest column of the ground truth $\boldsymbol{K}$ which is then deleted from $\boldsymbol{K}$ for the subsequent columns of $\hat{\boldsymbol{K}}$. This yields a lower bound to the true correlation between $K$ and $\hat{\boldsymbol{K}}$. More details will be given in an extended paper.

The model is generated 100 times with different parameters and we try to recover blindly $(\boldsymbol{K}, \boldsymbol{C})$ using Algorithm 1 starting from only one random initialization $\boldsymbol{C}_{0} \sim \mathcal{U}([0,1])$. For

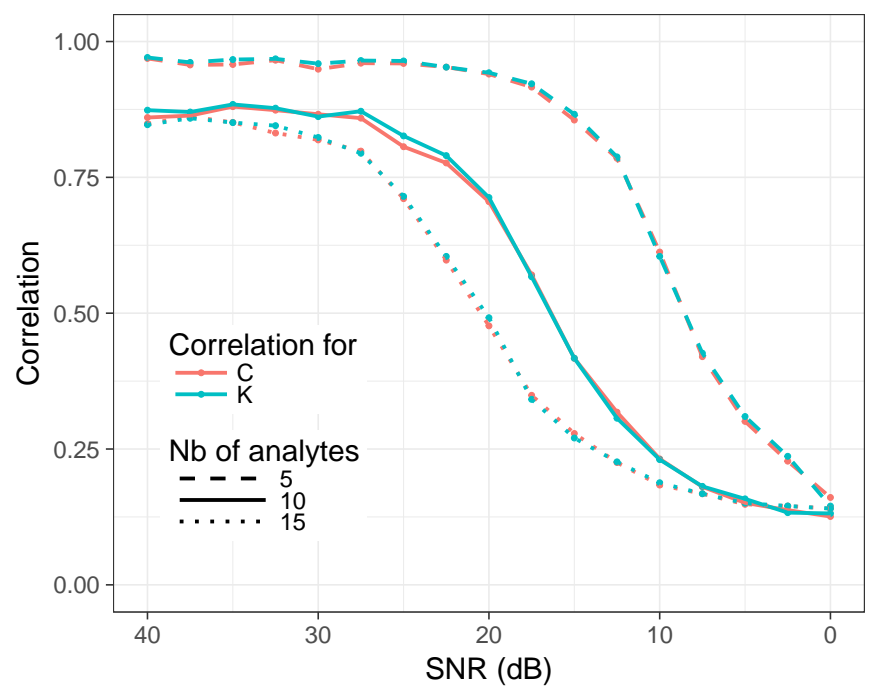

Figure 2: Correlation results for different SNR. From top to bottom, the number of analytes in the mixtures is 5,10 and 15 .

a given SNR, the correlation results, calculated as explained above, are averaged over the 100 trials.

Figure 2 shows that Algorithm 1 recovers correctly the signatures and their concentrations when the noise is sufficiently low. The fact that the performance is not exactly 1 in this case is explained by the non-convexity of the problem. Indeed, over the 100 realizations per SNR, the algorithm is highly expected to sometimes reach a local minimum which is not correlated with the ground truth, especially when we start from a single random initialization.

\section{CONCLUSION AND PROSPECTS}

We have described a mixture model based on the Langmuir isotherm for a multi-component gas, which can be applied for a type of chemical sensors used in an electronic nose. This model characterizes a non-linear relation between the concentration of each Volatile Organic Compound (VOC) in the mixture and their individual signature. This non-linearity makes it a quite challenging problem for a Blind Source Separation (BSS) issue.

For this purpose, we have derived conditions for joint identifiability of signatures and concentrations. We have also proposed a two-block coordinate descent algorithm in order to retrieve blindly these parameters. Performance has eventually been assessed in the presence of noise.

Further work will include experiments with real data. The assumption of masses knowledge also has to be relaxed. Finally, a mixture model depending on time derived from the system of differential equations (2) is currently being studied.

\section{ACKNOWLEDGMENT}

The authors would like to acknowledge Cyril Herrier and Thierry Livache from the start-up Aryballe Technologies for their highly valuable help in the understanding of the proposed model. 


\section{REFERENCES}

[1] K. Persaud and G. Dodd, "Analysis of discrimination mechanisms in the mammalian olfactory system using a model nose." Nature, vol. 299, no. 5881, pp. 352-355, 1982.

[2] T. C. Pearce, S. S. Schiffman, H. T. Nagle, and J. W. Gardner, Handbook of machine olfaction: electronic nose technology. John Wiley \& Sons, 2006.

[3] S. Madrolle, R. A. Ando, L. T. Duarte, R. Attux, C. Jutten, and P. Grangeat, "Méthodes de séparation de sources non linéaires pour des capteurs de gaz à oxyde métallique," in XXVIème Colloque Gretsi, 2017.

[4] A. Halperin, A. Buhot, and E. B. Zhulina, "On the hybridization isotherms of DNA microarrays: the Langmuir model and its extensions," Journal of Physics: Condensed Matter, vol. 18, no. 18 , p. S463, 2006.

[5] S. Brenet, A. John-Herpin, F. X. Gallat, A. Buhot, T. Livache, C. Herrier, T. Rousselle, and Y. Hou, "Development of a novel multiplexed optoelectronic nose for analysis of volatile organic compounds," in 2017 ISOCS/IEEE International Symposium on Olfaction and Electronic Nose (ISOEN), May 2017, pp. 1-3.

[6] E. Stenberg, B. Persson, H. k. Roos, and C. Urbaniczky, "Quantitative determination of surface concentration of protein with surface plasmon resonance using radiolabeled proteins," Journal of colloid and interface science, vol. 143, no. 2, pp. 513-526, 1991.

[7] E. Maillart, "Imagerie par résonance des plasmons de surface pour l'analyse simultanée de multiples interactions biomoléculaires en temps réel," Ph.D. dissertation, Université Paris SudParis XI, 2004.

[8] K. Huang, N. D. Sidiropoulos, and A. Swami, "Non-Negative Matrix Factorization Revisited: Uniqueness and Algorithm for Symmetric Decomposition," IEEE Transactions on Signal Processing, vol. 62, no. 1, pp. 211-224, Jan. 2014.

[9] P. Comon, "Independent component analysis, a new concept?" Signal processing, vol. 36, no. 3, pp. 287-314, 1994.

[10] A. Taleb and C. Jutten, "Nonlinear source separation: The post-nonlinear mixtures," in In: Proceedings of the ESANN'97. Citeseer, 1997.

[11] M. S. P. Kaare Brandt Petersen, The Matrix Cookbook. Technical University of Denmark, Nov. 2012.

[12] R. Byrd, P. Lu, J. Nocedal, and C. Zhu, "A Limited Memory Algorithm for Bound Constrained Optimization," SIAM Journal on Scientific Computing, vol. 16, no. 5, pp. 1190-1208, Sep. 1995. 